\title{
Théologiques
}

\section{Sens et spiritualité dans les pratiques professionnelles}

\section{Solange Lefebvre}

Volume 9, numéro 2, automne 2001

Sens et spiritualité dans les pratiques professionnelles

URI : https://id.erudit.org/iderudit/007291ar

DOI : https://doi.org/10.7202/007291ar

Aller au sommaire du numéro

Éditeur(s)

Faculté de théologie de l'Université de Montréal

ISSN

1188-7109 (imprimé)

1492-1413 (numérique)

Découvrir la revue

Citer cet article

Lefebvre, S. (2001). Sens et spiritualité dans les pratiques professionnelles.

Théologiques, 9(2), 3-5. https://doi.org/10.7202/007291ar d'utilisation que vous pouvez consulter en ligne.

https://apropos.erudit.org/fr/usagers/politique-dutilisation/ 


\section{Sens et spiritualité dans les pratiques professionnelles}

Solange LEFEBVRE

Faculté de théologie

Directrice du Centre d'études des religions

Université de Montréal

Les récentes années ont vu se multiplier les ateliers, conférences et best-sellers tous azimuts sur la crise du sens et la quête spirituelle. Les milieux universitaires s'y penchent de plus en plus, mais rarement regroupe-t-on des réflexions sur ces thèmes rattachées à plusieurs pratiques professionnelles. Théologiques présente ici de riches réflexions et analyses liées au milieu de la santé, à l'intervention psychosociale et à l'organisation du travail.

Jean Grondin nous offre en ouverture une réflexion sur la question du sens de la vie: "La question du sens de la vie présuppose que ce sens ne va pas, ne va plus de soi ", explique-t-il, rappelant que Nietzsche, le premier, et pourtant philosophe de l'absurde, forgea l'expression "sens de la vie ". Le sens existentiel ou spirituel de la vie ne va en effet plus de soi, mais il demeure une question. Une question qui rebondit aux divers tournants de l'existence : lors d'une maladie ou à l'approche de la mort, à l'occasion d'expériences professionnelles, sociales ou psychologiques, au cœur du travail, de plus en plus envahissant et pourtant souvent en crise de sens ... Comment cette question du sens existentiel ou spirituel se présente-t-elle à diverses occasions ? Et surtout, comment l'accueillir et l'intégrer, lorsqu'elle se pose à nous, dans les pratiques et les milieux professionnels ? 
Hubert Doucet (bioéthique) montre à cet égard les limites de l'éthique, dont l'importance s'accroît depuis les années 1960, en particulier dans les milieux de la santé. Après une phase théologique, suivie d'une approche juridique, objectivante et déductive, depuis les années 1980, un approfondissement du côté d'une phénoménologie de la maladie appelle à fonder l'éthique dans une recherche de sens, autour de la trame narrative de la personne et du souci d'autrui. Les infirmières orientent avec facilité leurs pratiques vers cette approche, puisque dès la fin du XIX ${ }^{\mathrm{e}}$ siècle, Florence Nightingale définissait la profession infirmière par le caring. La contribution de Chantale Cara et de Jacynthe Pepin (sciences infirmières), tout en évoquant cette pionnière de leur profession, rend compte de l'appréhension unitaire de la personne, indivisiblement corporelle, psychique et spirituelle, qui suscite depuis les années 1970 dans les approches infirmières une réflexion sur le sens et la spiritualité. Et cela après une période de marginalisation à la fois des femmes et des enjeux religieux dans les milieux de la santé. Une étude phénoménologique de Raymonde Cossette et Jacynthe Pepin clôt cette série d'articles liés au milieu de la santé, en rendant compte du paradoxe suivant : tout en voyant leur état s'aggraver, leurs déclins s'accumuler, des personnes âgées interrogées éprouvent le sentiment de croître et de trouver du sens à travers leurs déclins. De la sorte, cette étude s'inscrit dans les approches de la vieillesse vue comme une étape marquée par une expérience de transcendance.

L'article de François Lefebvre aborde les problèmes délicats que posent les convictions religieuses ou spirituelles particulières à l'intervention psychosociale, discutés surtout en Amérique du Nord. Il éclaire très bien les dilemmes rencontrés tant par les psychologues, les travailleurs sociaux que les intervenants dans le milieu de la santé ou scolaire. Selon l'anthropologie médicale, les convictions religieuses appartiennent à la culture des personnes et les repousser à la marge des pratiques professionnelles appauvrit l'interrelation. De plus, à l'égard des habituelles résistances des intervenants à intégrer la dimension spirituelle ou religieuses en particulier, il importe de rappeler l'importance d'une clarification de ses propres positions. Lefebvre montre bien l'étendue du spectre des attitudes à l'égard du religieux, en proposant ce qui lui semble le plus pertinent.

Une dernière partie traite des nouvelles questions posées par la spiritualité et la religion dans les organisations du travail. Christiane 
Demers et Sherazade Adib (changements organisationnels) analysent le thème de la spiritualité, présent surtout dans la littérature anglosaxonne sur le management. Est-ce le "nouveau paradigme " annoncé qui transformera les organisations ou une autre forme de mécanisme de contrôle social ? À travers la lecture des discours prospiritualités et d'autres plutôt critiques, ce texte aborde une question récente encore peu étudiée dans la littérature scientifique. Dans les diverses entreprises évoquées, il peut être résolument question de projets fondés sur la religion traditionnelle, sur une spiritualité plus large ou sur des valeurs fondamentales. Le texte suivant s'élabore justement à partir d'une étude empirique du point de vue des employés, assez rare selon Demers et Adib. Il est signé par trois auteurs, Solange Lefebvre, Jean-Philippe Perreault et Sherazade Adib. Des entrevues qualitatives effectuées en milieu québécois francophone révèlent que la privatisation de la religion, trait fondamental de la modernité sécularisée, marque fortement les individus. Les attentes de sens sont certes présentes en milieu de travail, mais surtout du côté d'une qualité de vie professionnelle et relationnelle. Les enjeux spirituels peuvent émerger, mais à la base, et on se défie des projets organisationnels qui voudraient les promouvoir.

Voilà plusieurs contributions qui composent un numéro précieux sur ces questions difficiles à clarifier. Mentionnons que l'article horsthème du théologien français Claude Geffré complète admirablement bien ce recueil, car il concerne la situation de deux grandes religions très présentes dans notre paysage contemporain, le christianisme et l'Islam, en relation avec la modernité critique. Voilà qui permet à ce numéro de la revue Théologiques de dépasser les discussions générales sur le religieux, le spirituel et la quête de sens, pour évoquer ces lieux toujours cruciaux de la conviction : les grandes traditions religieuses. 